\title{
Expression of hypoxia-related glucose transporters GLUT1 and GLUT3 in benign, malignant and non-neoplastic thyroid lesions
}

\author{
PAWEŁ JÓŹWIAK ${ }^{1}$, ANNA KRZEŚLAK ${ }^{1}$, LECH POMORSKI $^{2}$ and ANNA LIPIŃSKA ${ }^{1}$ \\ ${ }^{1}$ Department of Cytobiochemistry, University of Łódź, 90-236 Łódź; ${ }^{2}$ Department of General and Oncological Surgery, \\ Medical University of Łódź, 95-100 Zgierz, Poland
}

Received April 2, 2012; Accepted June 13, 2012

DOI: $10.3892 / \mathrm{mmr} .2012 .969$

\begin{abstract}
The enhancement of glucose metabolism in neoplastic cells is mediated by the overexpression of key glycolytic enzymes and glucose transporters (GLUTs). In particular, an increased expression of hypoxia-related GLUT1 and GLUT3 has been found in a variety of malignancies. The aim of this study was to examine the expression levels of GLUT1 and GLUT3 in benign and malignant thyroid tumors, as well as in non-neoplastic lesions. Analysis of the mRNA expression levels of solute carrier family 2, member 1 (SLC2A1) and solute carrier family 2, member 3 (SLC2A3) (genes coding GLUT1 and GLUT3, respectively) was performed by the real-time PCR method with fluorescent probes. GLUT1 and GLUT3 protein expression levels were determined in thyroid specimens by immunodetection after separation of proteins on $10 \%$ polyacrylamide slab gels and electrotransfer onto Immobilon-P membranes. The majority of papillary carcinoma samples showed a higher expression of GLUT1 and GLUT3 in comparison with follicular carcinoma cases and nonneoplastic thyroid lesions. A tendency towards an increased expression of GLUT1 and GLUT3 was observed in papillary carcinoma cases with more advanced disease stages. Moreover, a significant correlation was noted between the hypoxia-related GLUT1 and GLUT3 expression determined by both methods. In conclusion, our findings suggest that GLUT1 and GLUT3 play an important role in the pathology of thyroid glands.
\end{abstract}

\section{Introduction}

Since the early observation by Otto Warburg, it has been well known that tumor cells are characterized by an increased requirement for energy metabolism. Additionally, these cells, have a reduced ability to use mitochondrial oxidation and favor the conversion of pyruvate into lactate, despite the availability

Correspondence to: Professor Anna Lipińska, Department of Cytobiochemistry, University of Łódź, Pomorska 141/143, 90-236 Łódź, Poland

E-mail: annal@biol.uni.lodz.pl

Key words: glucose transporters 1 and 3, solute carrier family 2, members 1 and 3, thyroid lesions of oxygen. To compensate the inefficient extraction of energy from glucose, malignant cells have an at least a 20- to 30-fold higher rate of glycolysis than normal cells. This enhancement of glycolytic metabolism requires an increased rate of glucose uptake into tumor cells. The upregulation of glucose transport across the plasma membrane is mediated by a family of facilitative glucose transporter proteins (GLUTs), which differ regarding their tissue-specific distribution and affinity for glucose $(1,2)$. These structurally-related carriers contain 12 transmembrane spanning $\alpha$-helix domains with both the amino and carboxyl termini exposed on the cytoplasmic side of the plasma membrane (3).

The deregulation of glucose metabolism in cancer cells is predominantly mediated by oxygen-related transcription factors, such as the hypoxia-inducible factor 1 (HIF-1). HIF-1 induces a number of genes encoding glycolytic enzymes, erythropoietin, the tumor-associated carbonic anhydrases (CAs), CAIX and CAXII, vascular endothelial growth factor (VEGF), as well as the facilitative GLUT isoforms 1 and 3 (GLUT1 and GLUT3). Hypoxia-related GLUTs are characterized by low $\mathrm{Km}$ values and high affinity for glucose as compared to other members of the GLUT family. GLUT1 is mostly expressed in erythrocytes, placental cells and endothelial cells of the bloodbrain barrier, whereas GLUT3 is found mainly in the brain, placenta and other cell-types, with an intense requirement for glucose $(4,5)$. Previous studies have provided evidence of human malignancies expressing a higher level of GLUT1 and GLUT3 in comparison with normal cells. The overexpression of hypoxia-related GLUTs has been demonstrated in a variety of tumors, including lung, endometrium, breast, liver, kidney, head and neck, colon and oral squamous cell carcinomas (6-13). In general, the overexpression of GLUT1 and GLUT3 in different tumor types correlates with specific clinicopathological characteristics, malignant potential and poor prognosis. It has been suggested that the expression pattern of both transporters may be used as a prognostic factor of malignancy and progression, possibly assisting in the selection of patients requiring more aggressive therapy.

Some of the contradictory results concerning the level of GLUT1 and GLUT3 expression in thyroid lesions can be found in the literature. Therefore, the aim of this study was to clarify whether there is any difference in the expression of GLUT1 and GLUT3 between benign and malignant neoplasms and non-neoplastic thyroid lesions. 


\section{Materials and methods}

Patients and samples. The analyzed specimens were obtained from the Department of General and Oncological Surgery of the Medical University of Łódź. The material comprised samples from 73 patients, who underwent surgical resection due to nodular thyroid diseases. Thyroid specimens from patients were rapidly frozen and stored at $-80^{\circ} \mathrm{C}$ until needed. The histological diagnosis report of each patient was obtained from an experienced pathologist. Patient characteristics and specimens are presented in Table I. Typing and staging of tumors were carried out according to the system accepted by the International Union Against Cancer (UICC, 2010).

Isolation of cytoplasmic fraction. Pathological thyroid specimens were homogenized using a Potter's homogenizer in 10 volumes of ice-cold sucrose buffer $(0.25 \mathrm{M}$ sucrose, $5 \mathrm{mM}$ $\mathrm{MgCl}_{2}, 0.8 \mathrm{mM} \mathrm{KH}_{2} \mathrm{PO}_{4}, \mathrm{pH}$ 6.7) with $1 \mathrm{mM}$ phenylmethylsulfonyl fluoride (PMSF) to inhibit protease activity. The efficiency of homogenization was monitored by phase-contrast light microscopy. The supernatants obtained after homogenate centrifugation at $800 \mathrm{x} \mathrm{g}$ at $4^{\circ} \mathrm{C}$ for $10 \mathrm{~min}$ (sedimentation of nuclei) were considered as cytoplasmic fractions including cellular membranes and saved for further analysis.

RNA isolation and cDNA synthesis. Total RNA was isolated from frozen specimens by using Fenozol reagent (A\&A Biotechnology, Gdynia, Poland). The RNA quality was confirmed by electrophoresis on a $1.2 \%$ agarose gel with ethidium bromide staining and the $18 \mathrm{~S}$ and $28 \mathrm{~S}$ rRNA bands were visualized under ultraviolet light. The yields were quantified spectrophotometrically. RNA samples with a 260/280 nm ratio in the range 1.8-2.0 were saved for further analysis. cDNA was then synthesized from $2 \mu \mathrm{g}$ of total RNA using a RevertAid $^{\mathrm{TM}}$ First-Strand cDNA Synthesis kit (Fermentas Inc., Vilnius, Lithuania), following the manufacturer's instructions.

Quantitative real-time PCR. Quantitative real-time PCR with commercially available primers and fluorescent probes (TaqMan ${ }^{\circledR}$ Gene Expression Assay; Applied Biosystems ${ }^{\mathrm{TM}}$, Foster City, CA, USA) was employed to detect the expression of the target genes [solute carrier family 2, member 1 (SLC2A1) and solute carrier family 2, member 3 (SLC2A3)] encoding GLUT1 and GLUT3 in different types of thyroid lesions. The $G A P D H$ gene was used as the internal control. The assay numbers for these genes were as follows: Hs00892681_m1 and Hs00359840_m1, Hs99999905_m1.

Each PCR reaction was performed in a $10 \mu 1$ volume that included $5 \mu 1$ of $2 X$ TaqMan Universal PCR MasterMix (Applied Biosystems ${ }^{\mathrm{TM}}$ ), $4.5 \mu$ l of water-diluted cDNA tamplate and $0.5 \mu \mathrm{l}$ of TaqMan ${ }^{\circledR}$ Gene Expression assay consisting of a pair of unlabeled PCR primers and a TaqMan FAM ${ }^{\mathrm{TM}}$ fluorescent probe. The RT-qPCR reaction was carried out using the Mastercycler ep realplex (Eppendorf) under the following conditions: denaturation for $10 \mathrm{~min}$ at $95^{\circ} \mathrm{C}$ followed by 50 cycles of $15 \mathrm{sec}$ at $95^{\circ} \mathrm{C}, 1 \approx \min$ annealing and extension at $60^{\circ} \mathrm{C}$.

The $2^{-\triangle \mathrm{Ct}(\mathrm{Ctgene}-\mathrm{Ct} G A P D H)}$ method was used to estimate the relative gene expression levels in the analyzed samples. The $2^{-\Delta C \mathrm{t}}$ values were re-calculated into relative copy number values (number of $S L C 2 A 1$ or $S L C 2 A 3$ mRNA copies per 1,000 copies of GAPDH mRNA).

Western blot analysis. The protein samples were mixed with solubilizing buffer. Samples were not heated in a boiling water bath before SDS-PAGE but incubated for $15 \mathrm{~min}$ at room temperature in order to avoid GLUT protein aggregation. Denatured proteins of different types of thyroid lesions ( $30 \mu \mathrm{g}$ protein/lane) were resolved by electrophoresis on a $10 \%$ polyacrylamide slab gel with $0.1 \%$ SDS according to Laemmli (14) and electrotransferred onto Immobilon-P transfer membranes (Millipore, Bedford, MA, USA) by western blotting in Towbin's buffer (15). The quality of transfer was verified by Ponceau S staining before blocking the membrane. Then, the blots were incubated for $2 \mathrm{~h}$ at room temperature with the rabbit anti-human GLUT1 polyclonal antibodies in a 1:1000 dilution $\left(\mathrm{Abcam}^{\circledR}\right.$, Cambridge, UK) or mouse anti-human GLUT3 monoclonal antibodies in a 1:500 dilution, (Santa Cruz Biotechnology ${ }^{\circledR}$ Inc., Santa Cruz, CA, USA). Blots were washed 3 times with TBS-T ( $0.1 \%$ Tween- 20 in Tris-buffered saline, TBS) for $15 \mathrm{~min}$, and were incubated for $1 \mathrm{~h}$ at room temperature with horseradish peroxidase-labeled goat antirabbit or goat anti-mouse IgG antibodies, which were added at a dilution of 1:5000, (Santa Cruz Biotechnology Inc.). After extensive washing with TBS-T, the proteins were visualized on X-ray film by the enhanced chemiluminescence method. To confirm that the same amounts of proteins were loaded into each lane, the standard silver staining method was used for total protein identification on the gels (16). Gel-Pro Analyzer software version 3.0 (Media Cybernetics Inc., Bethesda, MD, USA) was used for densitometry analysis of the protein bands.

Statistical analysis. Statistical evaluation was performed using STATISTICA version 9.0 (StatSoft, Krakow, Poland). The non-parametric Mann-Whitney U test and Spearman rank analysis were applied. A p-value $<0.05$ was considered to indicate a statistically significant difference.

\section{Results}

Expression of GLUT1 and GLUT3 mRNA. mRNA levels of GLUT1 and GLUT3 were determined by real-time PCR in different thyroid specimens, such as non-neoplastic lesions i.e. nodular goiters (NGs) follicular adenomas (FAs), papillary carcinomas (PCs) and follicular carcinomas (FCs). The results of GLUT1 and GLUT3 gene expression in each type of thyroid lesions are presented in Table II and graphed in Fig. 1. The results indicate that $S L C 2 A 1$ gene (GLUT1) had a significantly higher expression in PCs than in non-neoplastic lesions $(\mathrm{p}<0.05)$ (Table II and Fig. 1A). In addition, a lower level of GLUT1 mRNA was observed in FCs in comparison with non-neoplastic, benign lesions and PCs $(\mathrm{p}<0.01)$. No statistically significant differences were found in GLUT1 mRNA levels between benign and non-neoplastic lesions. There were similar findings regarding the hypoxia-related $S L C 2 A 3$ gene (GLUT3). The data showed a higher expression level of GLUT3 mRNA in PC cases than in FCs $(\mathrm{p}<0.01)$ and nonneoplastic lesions $(\mathrm{p}<0.05)$ (Table II and Fig. 1B). Moreover, in the PC group, a tendency towards an increased expression of GLUT1 and GLUT3 mRNA with more advanced disease 
Table I. Characteristics of patients and surgically resected thyroid lesions.

\begin{tabular}{|c|c|c|c|}
\hline Diagnosis & Number of samples $(n=73)$ & Male:Female & Age (range) \\
\hline $\begin{array}{l}\text { Papillary carcinoma } \\
\text { Stage }\end{array}$ & 26 & $5: 21$ & $52.1(30-81)$ \\
\hline I & 6 & $1: 5$ & $41.8(30-66)$ \\
\hline II + III & 8 & $2: 6$ & $45.2(33-59)$ \\
\hline IV & 12 & $3: 9$ & $66.3(45-81)$ \\
\hline \multicolumn{4}{|l|}{ Lymph node metastasis } \\
\hline No & 12 & $3: 9$ & $58.1(33-81)$ \\
\hline Yes & 14 & $3: 11$ & $52.2(30-71)$ \\
\hline Follicular carcinoma & 6 & $2: 4$ & $63.5(32-78)$ \\
\hline Follicular adenoma & 11 & $2: 9$ & $57.7(32-64)$ \\
\hline Nodular goiter & 30 & $4: 26$ & $51.6(23-74)$ \\
\hline
\end{tabular}

Table II. Expression of SLC2A1 and SLC2A3 genes and their respective proteins, GLUT1 and GLUT3, in thyroid lesions.

\begin{tabular}{lcccc}
\hline & \multicolumn{2}{c}{ Gene $^{\mathrm{a}}$} & & \multicolumn{2}{c}{ Protein $^{\mathrm{b}}$} \\
\cline { 2 - 4 } Diagnosis & SLC2A1 & SLC2A3 & & GLUT1 \\
\hline Nodular goiter (NG) & $15.75 \pm 1.24$ & $30.85 \pm 5.13$ & $0.73 \pm 0.17$ & $2.42 \pm 0.36$ \\
Follicular adenoma (AF) & $23.32 \pm 1.88$ & $45.47 \pm 10.24$ & $0.92 \pm 0.27$ & $3.50 \pm 0.55$ \\
Papillary carcinoma (PC) & $25.22 \pm 2.23$ & $53.93 \pm 7.29$ & $1.23 \pm 0.23$ & $4.28 \pm 0.68$ \\
Follicular carcinoma (FC) & $10.95 \pm 1.35$ & $11.41 \pm 4.11$ & $0.30 \pm 0.06$ & $1.46 \pm 0.06$ \\
\hline
\end{tabular}

${ }^{\mathrm{a}}$ Copies of gene mRNA per 1,000 copies of GAPDH mRNA. ${ }^{\mathrm{b}}$ Integrated optical density. Values are expressed as the means \pm SEM. SLC2A1, solute carrier family 2, member 1; SLC2A3, solute carrier family 2, member 3; GLUT, glucose transporter.

stages was found (Fig. 1C and D). No statistically significant differences were noted in the expression of both genes in PC cases with or without metastasis to lymph nodes (Fig. 1E and F). A correlation was found between GLUT1 and GLUT3 mRNA expression (Spearman's rank analysis, $\mathrm{p}<0.001$ ).

GLUT1 and GLUT3 protein level. The expression of GLUT1 protein with the expected molecular masses in the range of 41-55 kDa (corresponding to its heterogeneously glycosylated species) was detected in all types of thyroid lesions (Fig. 2A). However, there were some differences in the level of GLUT1 expression between the various groups of thyroid lesions (Table II and Fig. 3A). The majority of the PC samples showed a higher immunoreactivity for GLUT1 in comparison with the non-neoplastic thyroid lesions $(\mathrm{p}<0.05)$ (Fig. 2A). The relative GLUT1 protein level was low in FCs compared to other types of lesions $(\mathrm{p}<0.01)$. A tendency towards an increased expression of GLUT1 was observed in the PC group with an elevated tumor stage (Fig. 3C). No obvious differences were noted regarding other histological features, such as the lymph node status (Fig. 3E).

The results of the GLUT3 expression in thyroid lesions determined by western blot analysis are shown in Fig. 2B. The highest expression of GLUT3 was associated with PCs as opposed to low GLUT3 immunoreactivity in FCs $(\mathrm{p}<0.01)$ and non-neoplastic lesions ( $\mathrm{p}<0.05)$ (Table II and Fig. 3B). No statistically significant differences were found in the GLUT3 protein level between non-neoplastic lesions (NGs) and the benign neoplasm group (FAs). Moreover, as observed in the GLUT1 expression patterns, the data showed a significantly higher GLUT3 protein level in stage IV than in stage I of PC (Fig. 3D). There were no statistically significant differences in GLUT3 expression in PC cases with or without metastasis to lymph nodes (Fig. 3F). There was a noted correlation between the GLUT1 and GLUT3 protein expression (Spearman's rank analysis, $\mathrm{p}<0.05$ ), whereas a significant correlation was noted between GLUT1 and GLUT3 expression levels determined by real-time PCR and those obtained by western blot analysis (Spearman's test analysis, $\mathrm{p}<0.01$ and $\mathrm{p}<0.05$, respectively).

\section{Discussion}

It is widely accepted that malignant cells enhance glucose metabolism. Such a high rate of glucose metabolism cannot be sustained without the upregulation of facilitative GLUTs $(1,2)$. In this study, using real-time PCR and western blot analysis, we investigated whether there is any difference in the expression of hypoxia-related GLUT1 and GLUT3 between benign and malignant neoplasms, as well as non-neoplastic thyroid lesions. 

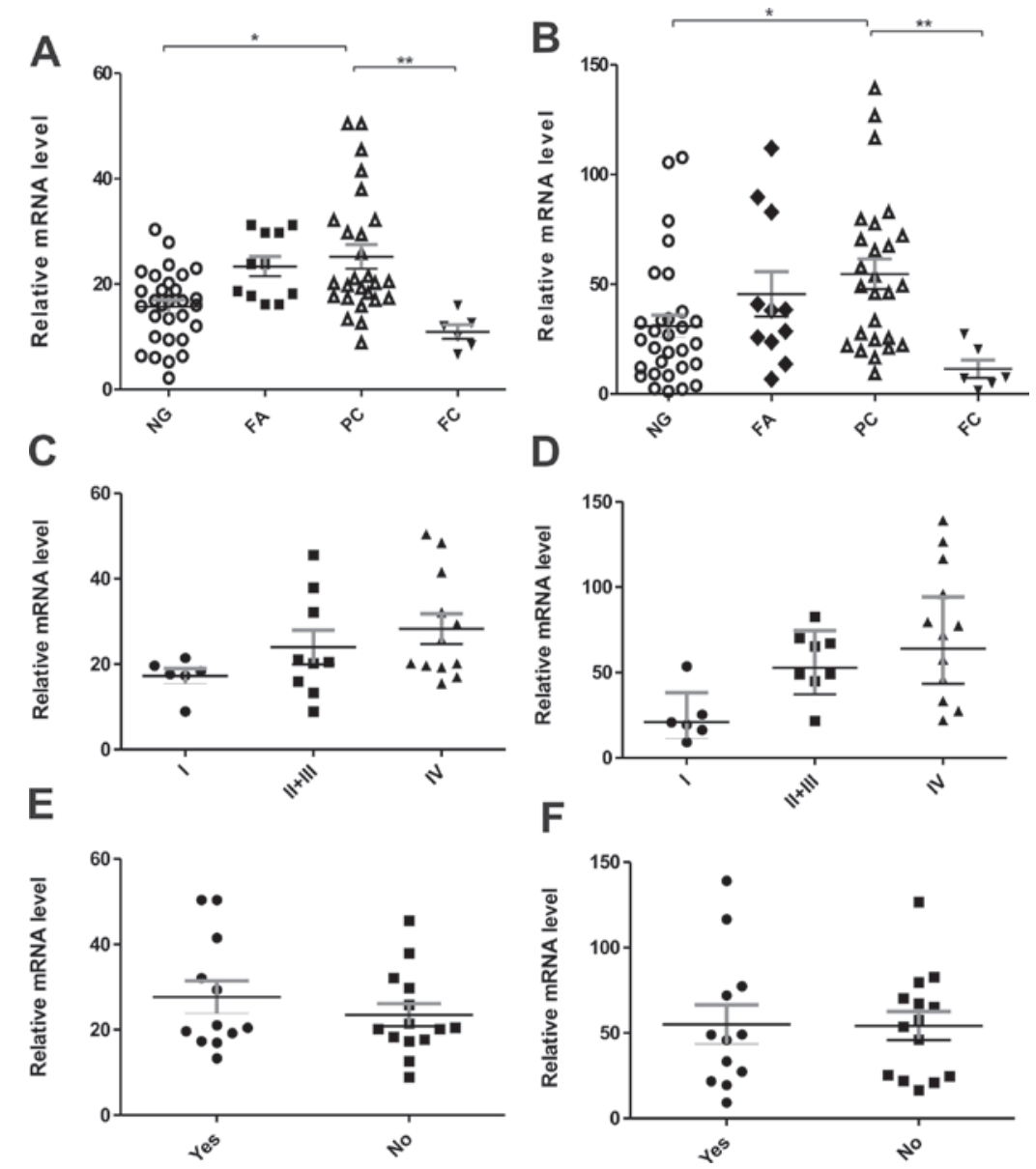

Figure 1. Real-time PCR analysis of (A, C and E) $S L C 2 A 1$ and (B, D and F) $S L C 2 A 3$. mRNA expression level in thyroid lesions via clinicopathological parameters: (A and B) diagnosis of nodular goiter (NG), follicular adenoma (FA), papillary carcinoma (PC) and follicular carcinoma (FC). (C and D) Tumor stages of PC. (E and F) PC with or without lymph node metastases. Error bars represent the means \pm SEM; U test; " $p<0.05 ;{ }^{* *} \mathrm{p}<0.01$.

$\mathrm{Mm}[\mathrm{kDa}]$

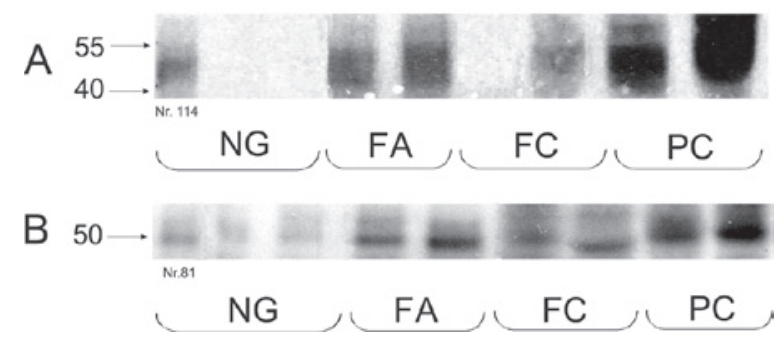

Figure 2. Analysis of (A) GLUT1 and (B) GLUT3 expression in thyroid pathological specimens by western blot analysis. Proteins of cytoplasmic fractions were resolved on $10 \%$ polyacrylamide slab gels ( $30 \mu \mathrm{g}$ protein/lane) and transferred onto Immobilon-P membranes. GLUT protein expression was detected using polyclonal and monoclonal antibodies against GLUT1 and GLUT3, respectively. NG, nodular goiter; FA, follicular adenoma; FC, follicular carcinoma; PC, papillary carcinoma. Specimens from patients Nr.114 and Nr.81 represent the reference samples for GLUT1 and GLUT3 densitometry analysis, respectively.

To date, GLUT1 expression in thyroid malignancies has mainly been investigated immunohistochemically. There are, however, some discrepancies between the results of these studies. Chandan et al (17) described negative GLUT1 staining in all $15(100 \%)$ examined cases with an unequivocal cytological diagnosis of PC. Kim et al (18) detected a positive expression of GLUT1 only in anaplastic carcinomas, which correlated with the impairment of p53 and p63 upregulation. Haber et al (19) reported a positive immunohistochemical expression of GLUT1 in both well- and poorly-differentiated thyroid carcinomas.

Our results, obtained by western blot analysis, showed a higher intensity of bands corresponding to GLUT1 in the majority of PC samples in comparison with non-neoplastic thyroid lesions. We also observed that the expression level of GLUT1 was significantly higher in PC than in FC cases, which is in agreement with the data previously reported (19-21). In our study on SLC2A1 (GLUT1) gene expression comparable findings were obtained.

There is little information in the literature regarding the GLUT3 expression in thyroid cancer. Ciampi et al (22) reported the most prevalent GLUT3 mRNA expression in well-differentiated thyroid papillary (NPA) and follicular (WRO) carcinoma cell lines. Another study showed slightly higher GLUT3 mRNA expression levels in thyroid cancer cells than in normal ones, although the results were not statistically significant (23). Contrary to the immunohistochemical analysis (21), we detected GLUT3 isoform expression both on the protein and mRNA levels. Our results revealed a higher level of GLUT3 in papillary than in FCs and non-neoplastic thyroid lesions. Moreover, we observed a tendency towards an 
A
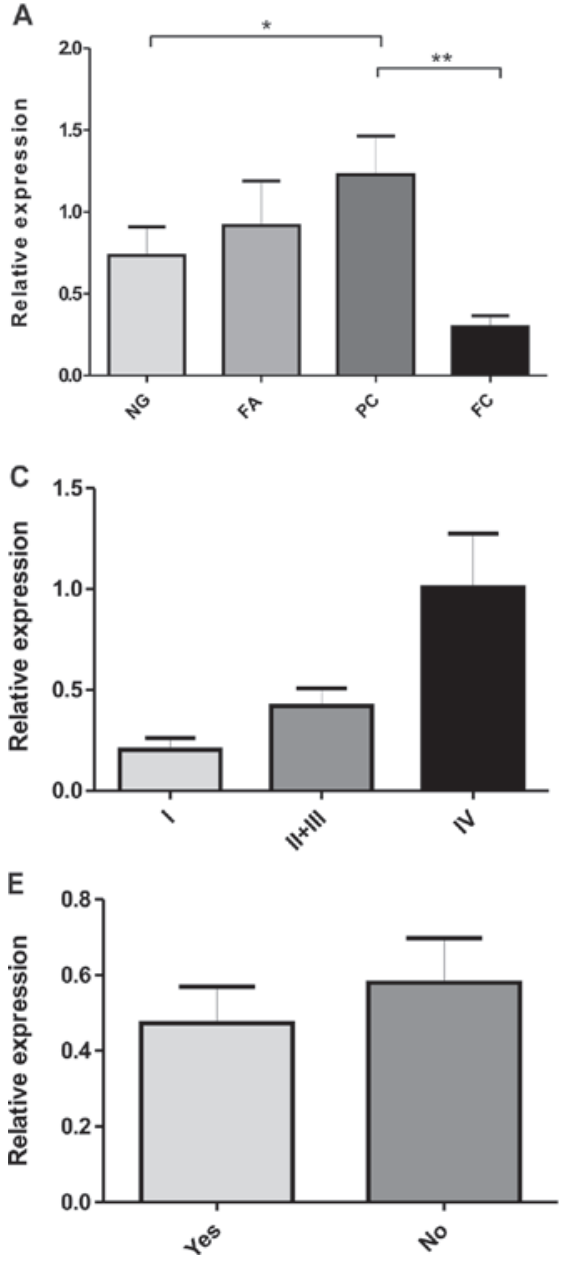
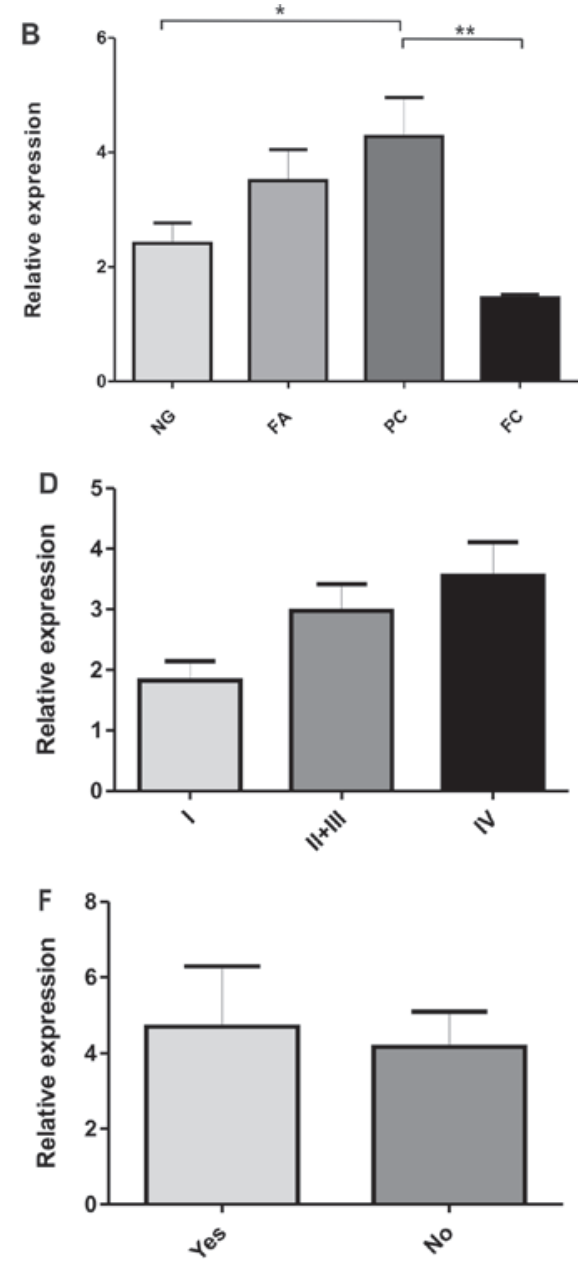

Figure 3. Densitometry analysis of (A, C and E) GLUT1 and (B, D and F) GLUT3 protein expression levels in thyroid lesions via clinicopathological parameters: (A and B) diagnosis of nodular goiter (NG), follicular adenoma (FA), papillary carcinoma (PC) and follicular carcinoma (FC). (C and D) Tumor stages of PC; (E and F) PC with or without lymph node metastases. The level of GLUT1 and GLUT3 expression was normalized by the reference sample (patient numbers 114 and 81, respectively) resolved by electrophoresis that made possible comparison intensity of the bands from different membranes. Error bars represent the mean \pm SEM; $\mathrm{U}$ test; ${ }^{*} \mathrm{p}<0.05 ;{ }^{* *} \mathrm{p}<0.01$.

increased expression of GLUT1 and GLUT3 in the PC group with a more advanced tumor stage.

The results of the present study suggest that the differences in GLUT1 and GLUT3 expression levels are associated with the histological type of thyroid carcinomas. Both hypoxia-related GLUT1 and 3 are involved in the progression of papillary thyroid carcinomas and may be added to a panel of biological thyroid carcinoma markers. For the first time in the literature we observed a close correlation between the overexpression of GLUT1 and GLUT3 proteins and the high levels of GLUT1 and GLUT3 mRNA in the same thyroid cancer specimens. However, in some neoplasm cases we were not able to detect the GLUT1 or GLUT3 positive band and the mRNA level was very low. A hypothesis that provides an acceptable explanation for these findings is that the expression of hypoxia-related GLUTs is further influenced by the microenvironment of tumor cells. Some authors have observed the most prominent GLUT1 expression around the necrotic areas or the hypoxic regions of tumors $(23,24)$. The study of Tomes et al $(25)$ on breast carcinomas showed that necrosis was usually associated with hypoxia and the co-expression of HIF1, CAIX and GLUT1. They also found that in cases of tumor epithelium without associated necrosis, GLUT1 was present at a low frequency. A similar phenomenon has been reported in thyroid malignancies, which usually show extensive GLUT1 immunostaining in the center of tumor cell groups or in perinecrotic areas $(20,21)$. In our study, we observed a correlation between GLUT1 and GLUT3 expression levels determined by both real-time PCR and western blot analysis. Our results suggest that hypoxia-related GLUTs in the thyroid may be regulated by common factors.

By contrast, the lack of hypoxia-related GLUTs in some examined samples suggests the possibility that other GLUT isoforms may be expressed. The expression of GLUT2, GLUT5 and GLUT10 has been reported in thyroid tissue $(21,26)$.

In conclusion, we presume that hypoxia-related GLUTs may play an important role in the glucose metabolism of thyroid neoplasms. Further investigations are required to clarify the exact regulation of hypoxia-related GLUT1 and GLUT3 in thyroid cancer cells.

\section{Acknowledgements}

This study was supported by the the University of Łódź (grant 505/0375), and is a 'Project co-funded by the European Union under the European Social Fund' and 'HUMAN-BEST INVESTMENT.' 


\section{References}

1. Airley RE and Mobasheri A: Hypoxic regulation of glucose transport, anaerobic metabolism and angiogenesis in cancer: novel pathways and targets for anticancer therapeutics. Chemotherapy 53: 233-256, 2007.

2. Ganapathy V, Thangaraju M and Prasad PD: Nutrient transporters in cancer: relevance to Warburg hypothesis and beyond. Pharmacol Ther 121: 29-40, 2009.

3. Joost H-G and Thorens B: The extended GLUT-family of sugar/ polyol transport facilitators: nomenclature, sequence characteristics, and potential function of its novel members (Review). Mol Membr Biol 18: 247-256, 2001.

4. Mueckler M: Facilitative glucose transporters. Eur J Biochem 219: 713-725, 1994.

5. Zhao F-Q and Keating AF: Functional properties and genomics of glucose transporters. Curr Genomics 8: 113-128, 2007.

6. Usuda K, Sagawa M, Aikawa H, Ueno M, Tanaka M, Machida Y, Zhao X-T, Ueda Y,Higashi K and Sakuma T: Correlation between glucose transporter-1 expression and 18F-fluoro-2-deoxyglucose uptake on positron emission tomography in lung cancer. Gen Thoroc Cardiovasc Surg 58: 405-410, 2010.

7. Goldman NA, Katz EB, Glenn AS, Weldon RH, Jones JG, Lynch U, Fezzari MJ, Runowicz CD, Goldberg GL and Charron MJ: GLUT1 and GLUT8 in endometrium and endometrial adenocarcinoma. Mod Pathol 19: 1429-1436, 2006.

8. Krzeslak A, Wojcik-Krowiranda K, Forma E, Jozwiak P, Romanowicz H, Bienkiewicz A and Brys M: Expression of GLUT1 and GLUT3 glucose transporters in endometrial and breast cancers. Pathol Oncol Res 18: 721-728, 2012.

9. Hernández F, Navarro M, Encinas JL, López Gutiérrez JC, López Santamaría M, Leal N, Martínez L, Patrón M and Tovar JA: The role of GLUT1 immunostaining in the diagnosis and classification of liver vascular tumors in children. J Pediatr Surg 40: 801-804, 2005

10. Suganuma N, Segade F, Matsuzu K and Bowden DW: Differential expression of facilitative glucose transporters in normal and tumour kidney tissues. BJU Int 99: 1143-1149, 2007.

11. Chandan VS, Faquin WC, Wilbur DC and Khurana KK: The ulility of GLUT-1 immunolocalization in cell blocks: An adjunct to the fine needle aspiration diagnosis of cystic squamous lesions of the head and neck. Cancer 108: 124-128, 2006.

12. Sakashita M, Aoyama N, Minami R, Maekawa S, Kuroda K, Shirasaka D, Ichihara T, Kuroda Y, Maeda S and Kasuga M: Glut1 expression in T1 and T2 stage colorectal carcinomas: its relationship to clinicopathological features. Eur J Cancer 37: 204-209, 2001.

13. Ayala FR, Rocha RM, Carvalho KC, Carvalho AL, da Cunha IW, Lourenço SV and Soares FA: GLUT1 and GLUT3 as potential prognostic markers ror oral squamous cell carcinoma. Molecules 15: $2374-2387,2010$
14. Laemmli UK: Cleavage of structural proteins during the assembly of the head of bacteriophage T4. Nature 227: 680-685, 1970.

15. Towbin H, Staehelin T and Gordon J: Electrophoretic transfer of protein from polyacrylamide gels to nitrocellulose sheets; procedure and some application. 1979. Biotechnology 24: 145-149, 1992.

16. Hochstrasser DF, Patchornik A and Merril CR: Development of polyacrylamide gels that improve the separation of proteins and their detection by silver staining. Anal Biochem 173: 412-423, 1988.

17. Chandan VS, Faquin WC, Wilbur DC and Khurana KK: The role of immunolocalization of CD57 and GLUT-1 in cell blocks in fine-needle aspiration diagnosis of papillary thyroid carcinoma. Cancer 108: 331-336, 2006.

18. Kim YW, Do IG and Park YK: Expression of the GLUT1 glucose transporter, p63 and p53 in thyroid carcinomas. Pathol Res Pract 202: 759-765, 2006.

19. Haber RS, Weiser KR, Pritsker A, Reder I and Burstein DE GLUT1 glucose transporter expression in benign and malignant thyroid nodules. Thyroid 7: 363-367, 1997.

20. Yasuda M, Ogane N, Hayashi H, Kameda Y, Miyagi Y, Iida T, Mori Y, Tsukinoki K, Minematsu T and Osamura Y: Glucose transporter-1 expression in the thyroid gland: clinicopathological significance for papillary carcinoma. Oncol Rep 14: 1499-1504, 2005.

21. Schönberger J, Rüschoff J, Grimm D, Marienhagen J, Rümmele P, Meyringer R, Kossmehl P, Hofstaedter F and Eilles C: Glucose transporter 1 gene expression is related to thyroid neoplasms with an unfavorable prognosis: an immunohistochemical study. Thyroid 12: 747-754, 2002.

22. Ciampi R, Vivaldi A, Romei C, Del Guerra A, Salvadori P, Cosci B, Pinchera A and Elisei R: Expression analysis of facilitative glucose transporters (GLUTs) in human thyroid carcinoma cell lines and primary tumors. Mol Cell Endocrinol 291: 57-62, 2008.

23. Brown RS, Goodman TM, Zasadny KR, Greenson JK and Wahl RL: Expression of hexokinase II and Glut-1 in untreated human breast cancer. Nucl Med Biol 29: 443-453, 2002.

24. Mori Y, Tsukinoki K, Yasuda M, Miyazawa M, Kaneko A and Watanabe Y: Glucose transporter type 1 expression are associated with poor prognosis in patients with salivary gland tumors. Oral Oncol 43: 563-569, 2007.

25. Tomes L, Emberley E, Niu Y, Troup S, Pastorek J, Strange K, Harris A and Watson PH: Necrosis and hypoxia in invasive breast carcinoma. Breast Cancer Res Treat 81: 61-69, 2003.

26. Matsuzu K, Segade F, Matsuzu U, Carter A, Bowden DW and Perrier ND: Differential expression of glucose transporters in normal and pathologic thyroid tissue. Thyroid 14: 806-812, 2004. 